\title{
Stability Analysis of SEIL Tuberculosis Epidemic Model with Logistic Growth in Susceptible Compartment
}

\author{
Joko Harianto $^{1 *}$ and Katarina Lodia Tuturop ${ }^{2}$ \\ ${ }^{1}$ Department of Mathematics, Faculty of Natural Sciences and Mathematics, Cenderawasih University, Papua, Indonesia \\ ${ }^{2}$ Department of Health Sciences, Faculty of Public Health, Cenderawasih University, Papua, Indonesia

\begin{abstract}
This article discusses modifications to the SEIL model that involve logistical growth. This model is used to describe the dynamics of the spread of tuberculosis disease in the population. The existence of the model's equilibrium points and its local stability depends on the basic reproduction number. If the basic reproduction number is less than unity, then there is one equilibrium point that is locally asymptotically stable. The equilibrium point is a disease-free equilibrium point. If the basic reproduction number ranges from one to three, then there are two equilibrium points. The two equilibrium points are disease-free equilibrium and endemic equilibrium points. Furthermore, for
\end{abstract} \\ this case, the endemic equilibrium point is locally asymptotically stable.
}

Keywords: logistic growth; local stability; equilibrium point; endemic equilibrium point

\section{INTRODUCTION}

Death cases of tuberculosis between 2000 and 2015 decreased to $22 \%$. According to a WHO report in 2016, this disease still ranks as the 1oth highest death cause globally. The incidence of transmission of tuberculosis in 2016 globally was 10.4 million cases. The incidence is equivalent to 120 cases per 100,000 population. India, Indonesia, China, the Philippines, and Pakistan are the five countries with the highest tuberculosis cases. Therefore, attention to tuberculosis is still a priority in global health problems and is even one of the SDGs (Sustainability Development Goals).

Control of this disease transmission problem needs to do with predictive data on the number of infected cases in the future. Estimating the number of cases of prolonged tuberculosis infection requires a mathematical approach. Mathematical models and numerical simulations are approaches that are easy to manage, inexpensive, relatively fast, and quite productive. Mathematical models need to be used to explain and predict the behaviour of the spread of tuberculosis. Waaler et al. first introduced the model. They classified the population into three sub-populations and compiled a model based on the spread of tuberculosis (Waaler, Geser \& Andersen, 1962). The tuberculosis spread model developed by Revelle et al. involves a non-linear system of ordinary differential equations. They first introduce tuberculosis transmission, depending on its prevalence (Revelle, Lynn \& Feldmann, 1967). Castillo et al. described several mathematical models for the dynamics of tuberculosis transmission. These models discuss two infected classes, the distributed delayed tuberculosis model, the exogenousreinfection tuberculosis, and the age-structured tuberculosis model. They analyse each model's stability by involving basic reproduction numbers (Castillo-Chavez \& Feng, 1997). Castilo and Song reviewed various models of tuberculosis and developed a different concept for reconstructing the tuberculosis model (Castillo-Chavez \& Song, 2004). The dynamics model of tuberculosis transmission considers the factors of disease progression, treatment, vaccination, immigration, and others presented in articles (Jia et. al., 2008; Athithan \& Ghosh, 2013; Wangari, Trauer \& Stone, 2018). Over the years, mathematical models have been continuously developed to study the transmission dynamics of tuberculosis. Zhao et al. investigated the role of age in

*Corresponding author's e-mail: joharijpr88@gmail.com 
tuberculosis transmission in mainland China and found that the BCG vaccine is only useful for young people (Zhao, Li \& Yuan, 2017). Then, Moualeu et al. developed a dynamic model of tuberculosis transmission and applied it to existing data in Cameroon (Moualeu et al., 2015).

The theoretical framework related to optimal control in the model also has been used to propose control tuberculosis measures. Jung et al. first introduced an optimal control strategy in a dynamic tuberculosis disease transmission (Jung, Lenhart \& Feng, 2002). Then, Whang et al. applied the model to the spread of tuberculosis in the Republic of Korea (Whang, Choi \& Jung, 2011). Kim et al. also used the model to spread disease in the Philippines (Kim, de los Reyes \& Jung, 2018). Choi and Jung also provided practical policies by explaining the comparison of government budgets related to tuberculosis control with optimal control results (Choi \& Jung, 2014). Villasin et al. describes a dynamic model of tuberculosis transmission in the Philippines. The model proposed by Villasin et al. consider the effect of vaccination and estimate parameters from current data. Some parameters are used to investigate intervention strategies. Villasin et al. concluded that enhancing partial immunity, maximising treatment duration, and rapidly detecting new cases will effectively reduce the incidence and prevalence of tuberculosis than increasing vaccine coverage (Villasin \& Lao, 2017).

All discussions about dynamic models of tuberculosis that have been described above involve exponential growth in the population. Therefore, it is necessary to study these models by applying exponential growth in the population. This article discusses a dynamic model of tuberculosis transmission adapted from Kim et al. (Kim, de los Reyes \& Jung, 2018). This model was modified to explain the dynamics of the spread of tuberculosis involving logistical growth factors.

\section{MATERIALS AND METHOD}

The method used in this study is a literature review. The author read several reputable international articles related to the tuberculosis model. The author later developed the concepts found in these articles.

The first step in this article's discussion is to read all items related to the tuberculosis model. The next step identifies the concepts developed in the tuberculosis model. They are then forming a tuberculosis model according to this concept. The model constructed is then analysed using the theory in the book written by Perko et al. (2001). Model analysis includes an equilibrium point, the existence of the basic production number, and the equilibrium point's stability. The final section of the discussion gives a numerical simulation to check the analysis results obtained.

\section{RESULT AND DISCUSSION}

\section{A. Model Formulation}

The model presented in this article is a modification of the previous model discussed in Kim et al. article. The total population in time $t$ denoted by $N(t)$. The population is divided into five epidemiological classes, i.e. the susceptible class, the high-risk class, the infected class and the low-risk class. The number of people in the susceptible class in time $t$ denoted by $S(t)$. The number of people in the class of exposed to high-risk in time $t$ denoted by $E(t)$. The number of population in the infected class in time $t$ denoted by $I(t)$. The number of people in the class of exposed to low-risk in time $t$ denoted by $L(t)$. Birth in all classes is assumed to enter the susceptible class directly, and its growth follows exponential growth (Kim, de los Rayes \& Jung, 2018). The assumptions that distinguish the model from the models discussed in this article are that the population growth in the susceptible class is assumed to follow logistical growth. This model also assumes that the class of infected or exposed to low or highrisk does not contribute to births and deaths in the susceptible class. The modified tuberculosis disease epidemic model is based on these two assumptions given in the following system of non-linear differential equations:

$$
\left.\begin{array}{l}
\frac{d S}{d t}=b S\left(1-\frac{S}{K}\right)-\beta S I \\
\frac{d E}{d t}=\beta S I-(\alpha+\gamma+\mu) E+p r I \\
\frac{d I}{d t}=\gamma E-(\mu+r+d) I \\
\frac{d L}{d t}=(1-p) r I+\alpha E-\mu L
\end{array}\right\}
$$

with a non-negative initial value and $N(t)=S(t)+E(t)+$ $I(t)+L(t)$. The parameter $b$ is the intrinsic rate of the susceptible class. $K$ is the carrying capacity of the susceptible class in an area. The natural death rate for all classes is denoted by $\mu$. Susceptible individuals can expose to tuberculosis disease through contact with individuals 
infected with tuberculosis disease with transmission at the rates $\beta$. The rate of treatment in the high-risk class is denoted by $\alpha$. Some patients who are not maximal in receiving treatment in the high-risk class will develop into infected patients who are admitted to the infectious class at the rates $\gamma$. Treatment to the infected class at the rates $r$. Then some of the patients in that class their regular treatment at the rates $(1-p) r$ with $0<p<1$. Recovered patients are transferred to low-risk exposure classes because treatment cannot eradicate tuberculosis in the patient's body. Therefore, recovered patients and individuals exposed to low-risk were classified into one class only, namely the low-risk exposure class. The death rate of tuberculosis disease denoted by $d$. All parameters used in this model are positive.

Let $X(t)=(S(t), E(t), I(t), L(t)), X^{\prime}(t)=\left(\frac{d S}{d t}, \frac{d E}{d t}, \frac{d I}{d t}, \frac{d L}{d t}\right)$, $f=\left(f_{1}, f_{2}, f_{3}, f_{4}\right)$ and $f: X \rightarrow X^{\prime}$ then the system (1) can write in the following system:

$$
X^{\prime}(t)=f(X(t)
$$

with

$$
\begin{aligned}
& f_{1}(X)=b S\left(1-\frac{S}{K}\right)-\beta S I \\
& f_{2}(X)=\beta S I-(\alpha+\gamma+\mu) E+p r I \\
& f_{3}(X)=\gamma E-(\mu+r+d) I \\
& f_{4}(X)=(1-p) r I+\alpha E-\mu L
\end{aligned}
$$

and initial values $X(0)=\left(S_{0}, E_{0}, I_{0}, L_{0}\right) \in\{0\} \cup R_{+}^{4}$.

Theorem 1.

System (2) with non-negative initial conditions has a unique non-negative solution $X(t)$ and finite. The solution $X(t)$ is non-negative.

Proof.

Equation (3) - (6) is a continuously differentiable function. According to the theorem of existence solution (Perko, 2001), system (2) has a unique solution $X(t)$ for every $t$ nonnegative. The implicit solution of the first equation in the system (2) can obtain using the integration factor method (Li et al., 2017) and (Mengistu \& Witbooi, 2019). The following solutions $S(t)$ obtained

$$
S(t)=S_{0} \exp \left(\int_{0}^{t}\left(b-\frac{b S(u)}{K}-\beta I(u)\right) d u\right) \geq 0, \forall t \geq 0
$$

Solution $S(t)$ is non-negative for every $t$ non-negative. Analogous to the solution $S(t)$ specified above, it can show that the solution $E(t), I(t)$ and $L(t)$ is also non-negative. If the total population changes with time $t$, that is

$N(t)=S(t)+E(t)+I(t)+L(t)$

$\frac{d N}{d t}=\frac{d S}{d t}+\frac{d E}{d t}+\frac{d I}{d t}+\frac{d L}{d t}$

$$
=b S\left(1-\frac{S}{K}\right)-\mu(E+I+L)-d I<b S\left(1-\frac{S}{K}\right)-\mu N
$$

then there exist $M=\max \left\{S_{0}, K\right\}$ such that $\lim _{t \rightarrow \infty} \sup N(t) \leq \frac{b M}{\mu}$. It shows that the solutions are limited. So, Theorem 1 is proven.

\section{B. Equilibrium and Basic Reproduction Number}

The equilibrium point of system (2) is the solution of system (2), which does not change with time (constant solution). The constant solution of the system (2) is obtained from the following equation.

$$
X^{\prime}(t)=f(X(t))=0
$$

Let $P=\left(S^{*}, E^{*}, I^{*}, L^{*}\right)$ is the equilibrium point for the system (2) with

$$
\begin{aligned}
f_{1}(X)=0 & \Rightarrow & b S^{*}\left(1-\frac{S^{*}}{K}\right)-\beta S^{*} I^{*}=0 \\
f_{2}(X)=0 & \Rightarrow & \beta S^{*} I^{*}-(\alpha+\gamma+\mu) E^{*}+p r I^{*}=0 \\
f_{3}(X)=0 & \Rightarrow & \gamma E^{*}-(\mu+r+d) I^{*}=0 \\
f_{4}(X)=0 & \Rightarrow & (1-p) r I^{*}+\alpha E^{*}-\mu L^{*}=0
\end{aligned}
$$

From Equation (9), we obtained

$$
E^{*}=\frac{n}{\gamma} I^{*}
$$

If Equation (11) substituted in Equation (8), we have

$$
\left(\beta S^{*}-\frac{m n}{\gamma}+p r\right) I^{*}=0
$$

with $m=\alpha+\gamma+\mu$ and $n=\mu+r+d$.

Based on Equation (12), the value of $I^{*}$ can divide into two cases, i.e. $I^{*}=0$ and $I^{*} \neq 0$.

For the first case, If $I^{*}=0$, then $E^{*}=L^{*}=0$ obtained. If $I^{*}=0$ substituted to Equation (7), then $b S^{*}\left(1-\frac{S^{*}}{K}\right)=0$, so that we obtained $S^{*}=0$ or $S^{*}=K$. Thus, for the case $I^{*}=0$, there exist two equilibrium points for the system (2), i.e. $P_{0}=(0,0,0,0)$ and $P_{1}=(K, 0,0,0)$. The equilibrium point $P_{0}$ 
is a trivial solution for the system (2). The equilibrium point and

$P_{0}$ stable means that the human population will eventually disappear. Thus, the equilibrium point $P_{0}$ not discussed. The equilibrium point $P_{1}$ defined as a condition with a population that is susceptible to tuberculosis disease as much $K$, and no one exposes to a low, high, or even infection risk. The diseasefree equilibrium $P_{1}$ means that no patient is infected, no patient exposed to high-risk, and no patient is exposed to low risk (zero value). For the second case, if $I^{*} \neq 0$ then from Equation (12) it must be

$$
\beta S^{*}-\frac{m n}{\gamma}+p r=0,
$$

So that we obtained

$$
S^{*}=\frac{m n-\gamma p r}{\beta \gamma}
$$

with $m=\alpha+\gamma+\mu$ and $n=\mu+r+d$. It is clear that $S^{*}>0$, because $m n$ contains the term $\gamma r$ and $0<p<1$. If Equation (13) is substituted to Equation (7), we have

$$
I^{*}=\frac{K \gamma \beta b+b \gamma p r-b m n}{\beta^{2} K \gamma}
$$

If Equation (11) substituted to Equation (10), we obtained

$$
L^{*}=\left(\frac{\gamma(1-p) r+\alpha n}{\mu \gamma}\right) I^{*}
$$

So, for the case $I^{*} \neq 0$, we obtained $P^{*}=\left(S^{*}, E^{*}, I^{*}, L^{*}\right)$ with

$$
\begin{gathered}
S^{*}=\frac{m n-\gamma p r}{\beta \gamma} \\
E^{*}=\frac{n}{\gamma} I^{*} \\
I^{*}=\frac{K \gamma \beta b+b \gamma p r-b m n}{\beta^{2} K \gamma} \\
L^{*}=\left(\frac{\gamma(1-p) r+\alpha n}{\mu \gamma}\right) I^{*} .
\end{gathered}
$$

The basic reproduction number is the average number of new infection cases caused by an infected patient coming into contact with someone from a susceptible class. The basic reproduction number can be determined using the next generation method (Driessche van den, 2017). Based on the equations in the system (1), we obtained:

$$
F=\left[\begin{array}{cc}
0 & \beta K+p r \\
0 & 0
\end{array}\right]
$$

$$
V=\left[\begin{array}{cc}
m & 0 \\
-\gamma & n
\end{array}\right]
$$

The next-generation matrix for the system (1) that is

$$
F V^{-1}=\left[\begin{array}{cc}
\frac{\beta K \gamma+\gamma p r}{m n} & \frac{\beta K+p r}{n} \\
0 & 0
\end{array}\right]
$$

The basic reproduction number for the system (1) is the spectral radius of $F V^{-1}$, that is

$$
\Re_{0}=\rho\left(F V^{-1}\right)=\frac{\gamma \beta K+\gamma p r}{m n}
$$

The relationship between the basic reproduction number and $I^{*}$ in Equation (14) can write as follows:

$$
I^{*}=\frac{b m n}{K \gamma \beta^{2}}\left(\Re_{0}-1\right),
$$

So that we obtain

$$
E^{*}=\frac{b m n^{2}}{K \gamma^{2} \beta^{2}}\left(\Re_{0}-1\right),
$$

and

$$
L^{*}=\frac{((1-p) r \gamma+\alpha n) b m n}{K \mu \gamma^{2} \beta^{2}}\left(\Re_{0}-1\right),
$$

The existence of a disease-free and endemic equilibrium point of system (1) in relation to the basic reproduction number it can be concluded that if $\Re_{0}<1$, then there is one equilibrium point, namely the disease-free equilibrium point $P_{1}=(K, 0,0,0)$. Conversely, if $\mathfrak{R}_{0}>1$, then there are two equilibrium points, namely the disease-free equilibrium point $P_{1}=(K, 0,0,0)$ and the endemic equilibrium point $P^{*}=\left(S^{*}, E^{*}, I^{*}, L^{*}\right)$.

\section{Local Stability Analysis}

This subsection discusses the analysis of the local stability of the disease-free and endemic equilibrium points of the system (1). Equations (3), (4), and (5) do not depend on $L$ so that the fourth equation does not need to be analysed to simplify calculations. System (1) can reduce to

$$
\left.\begin{array}{l}
\frac{d s}{d t}=b S\left(1-\frac{S}{K}\right)-\beta S I \\
\frac{d E}{d t}=\beta S I-(\alpha+\gamma+\mu) E+p r I \\
\frac{d I}{d t}=\gamma E-(\mu+r+d) I
\end{array}\right\}
$$


The disease-free and endemic equilibrium point's local stability is determined using the eigenvalues of the Jacobian System matrix (16). In general, the Jacobian matrix from the system (16) can write as follows:

$$
J=\left[\begin{array}{ccc}
b-\frac{2 b S}{K}-\beta I & 0 & -\beta S \\
\beta I & -m & \beta S+p r \\
0 & \gamma & -n
\end{array}\right]
$$

Therefore, the local stability analysis of the system's equilibrium points (16) can present in the following theorem. Theorem 2.

If $\Re_{0}<1$, then the disease-free equilibrium $P_{1}=(K, 0,0,0)$ of system (1) is locally asymptotically stable and $P_{1}$ unstable if $\Re_{0}>1$ with $\Re_{0}=\frac{\gamma \beta K+\gamma p r}{m n}$.

Proof.

The Jacobian matrix at the equilibrium point $P_{1}=(K, 0,0,0)$ that is

$$
J\left(P_{1}\right)=\left[\begin{array}{ccc}
-b & 0 & -\beta K \\
0 & -m & \beta K+p r \\
0 & \gamma & -n
\end{array}\right]
$$

The characteristic equation of $J\left(P_{1}\right)$, that is

$$
(\lambda+b)\left(\lambda^{2}+(m+n) \lambda+m n-\gamma \beta K-p r\right)=0
$$

If Equation (17) involves the basic reproduction number, we obtained

$$
(\lambda+b)\left(\lambda^{2}+(m+n) \lambda-m n\left(\Re_{0}-1\right)\right)=0
$$

From Equation (18), one of the eigenvalues of $J\left(P_{1}\right)$ obtained, i.e. $\lambda_{1}=-b<0 . \lambda_{1}$ is negative because $b$ is a positive parameter. The other eigenvalues of $J\left(P_{1}\right)$ depends on the basic reproduction number. If $\Re_{0}<1$, then based on the Routh-Hurwitz criterion, all of the eigenvalues of $J\left(P_{1}\right)$ negative. Based on the theorem of stability (Perko, 2001), if the real part of all eigenvalues of the Jacobian matrix $J\left(P_{1}\right)$ negative, then the equilibrium point $P_{1}=(K, 0,0,0)$ is locally asymptotically stable. Conversely, if $\Re_{0}>1$, then there is a positive eigenvalue of the Jacobian matrix $J\left(P_{1}\right)$; as a result, the equilibrium point $P_{1}=(K, 0,0,0)$ is unstable.

Theorem 3.

If $1<\mathfrak{R}_{0}<3$, then there exist the endemic equilibrium point $P^{*}=\left(S^{*}, E^{*}, I^{*}, L^{*}\right)$ of the system (1) which is locally asymptotically stable with
$\mathfrak{R}_{0}=\frac{\gamma \beta K+\gamma p r}{m n}$,

$S^{*}=\frac{m n-\gamma p r}{\beta \gamma}>0$,

$I^{*}=\frac{b m n}{K \gamma \beta^{2}}\left(\Re_{0}-1\right)$,

$E^{*}=\frac{b m n^{2}}{K \gamma^{2} \beta^{2}}\left(\Re_{0}-1\right)$,

$L^{*}=\frac{((1-p) r \gamma+\alpha n) b m n}{K \mu \gamma^{2} \beta^{2}}\left(\Re_{0}-1\right)$,

$m=\alpha+\gamma+\mu$ and $n=\mu+r+d$.

Proof.

Clearly that if $\Re_{0}>1$, then there exist the endemic equilibrium point $P^{*}=\left(S^{*}, E^{*}, I^{*}, L^{*}\right)$ of the system (1). The Jacobian matrix at the equilibrium point $P^{*}=\left(S^{*}, E^{*}, I^{*}\right)$, that is

$$
J\left(P^{*}\right)=\left[\begin{array}{ccc}
-\frac{b S^{*}}{K} & 0 & -\beta S^{*} \\
\beta I^{*} & -m & \frac{m n}{\gamma} \\
0 & \gamma & -n
\end{array}\right]
$$

The characteristic equation of $J\left(P^{*}\right)$ that is

$$
\lambda^{3}+a_{1} \lambda^{2}+a_{2} \lambda+a_{3}=0
$$

with

$$
\begin{gathered}
a_{1}=\frac{b S^{*}}{K}+m+n \\
a_{2}=\frac{b S^{*}}{K}(m+n) \\
a_{3}=\beta^{2} S^{*} I^{*}
\end{gathered}
$$

It is clear that $a_{1}>0, a_{2}>0$, and $a_{3}>0$. We have

$$
\begin{gathered}
a_{1} a_{2}-a_{3}=\left(\frac{b S^{*}}{K}+m+n\right)\left(\frac{b m S^{*}}{K}+\frac{b n S^{*}}{K}\right)-\beta^{2} \gamma S^{*} I^{*} \\
=\frac{b^{2} m S^{* 2}}{K^{2}}+\frac{b^{2} n S^{* 2}}{K^{2}}+\frac{b m^{2} S^{*}}{K}+\frac{b n^{2} S^{*}}{K}+\frac{2 b m n S^{*}}{K} \\
-\frac{b m n S^{*}}{K}\left(\Re_{0}-1\right)
\end{gathered}
$$

$=\frac{b^{2} m S^{* 2}}{K^{2}}+\frac{b^{2} n S^{* 2}}{K^{2}}+\frac{b m^{2} S^{*}}{K}+\frac{b n^{2} S^{*}}{K}+\frac{3 b m n S^{*}}{K}-\frac{b m n S^{*} \Re_{0}}{K}$ $=\frac{b^{2} m S^{* 2}}{K^{2}}+\frac{b^{2} n S^{* 2}}{K^{2}}+\frac{b m^{2} S^{*}}{K}+\frac{b n^{2} S^{*}}{K}+\frac{b m n S^{*}}{K}\left(3-\Re_{0}\right)$

As observed in Equation (18), we know that $a_{1} a_{2}-a_{3}>0$ can hold if $\Re_{0}<3$. Thus, based on the Routh-Hurwitz criterion, the real part of all eigenvalues are negative if $\Re_{0}<3$. Meanwhile, the conditions $\mathfrak{R}_{0}>1$ must also hold to 
ensure the existence of an endemic equilibrium point. Thus, the endemic equilibrium point $P^{*}=\left(S^{*}, E^{*}, I^{*}, L^{*}\right)$ exist and locally asymptotically stable if $1<\Re_{0}<3$.

\section{Numerical Simulation}

In the following, the system (1) discusses numerically to support the analysis results that have been obtained. The numerical simulation in the system (1) carried out by reviewing three cases, i.e., simulation for cases $\mathfrak{R}_{0}<1, \quad 1<$ $\Re_{0}<3$ and $\Re_{0}>3$. The parameters used for each case given in the following table.

Table 1. Parameter Values for Cases $\Re_{0}<1$

\begin{tabular}{c|c|c}
\hline Parameter & $\begin{array}{c}\text { Estimated } \\
\text { (per year) }\end{array}$ & Source \\
\hline$b$ & 0,04 & (Kim et al., 2018) \\
$\beta$ & $1,5 \times 10^{-7}$ & (Mengistu \& Witbooi, 2019) \\
$\mu$ & 0,02 & (Kim et al., 2018) \\
$\alpha$ & 0,2 & (Kim et al., 2018) \\
$\gamma$ & 0,023 & (Mengistu \& Witbooi, 2019) \\
$p$ & 0,2 & (Kim et al., 2018) \\
$r$ & 0,546 & (Mengistu \& Witbooi, 2019) \\
$d$ & 0,17 & (Mengistu \& Witbooi, 2019) \\
$K$ & $5 \times 10^{7}$ & Assumed \\
$S_{0}$ & $3,8 \times 10^{7}$ & (Mengistu \& Witbooi, 2019) \\
$E_{0}$ & $1,19 \times 10^{7}$ & (Mengistu \& Witbooi, 2019) \\
$I_{0}$ & $3,73 \times 10^{5}$ & (Mengistu \& Witbooi, 2019) \\
$L_{0}$ & $2,18 \times 10^{7}$ & (Mengistu \& Witbooi, 2019) \\
\hline
\end{tabular}

Based on the values of the parameters in Table 1, we have $\Re_{0}=0,98$. The numerical solution of system (1) for this case is given in Figure 1.

Figure 1 shows that the number of population in the susceptible class $S(t)$ initially decreases, then rises towards the number $5 \times 10^{7}$ and stabilises at that number. In contrast, the population in the low-risk class $L(t)$ initially grows, then falls towards zero and stabilises at zero. The population in the high-risk class $E(t)$ and the infected class $I(t)$ drops to zero and stabilises at that number. It means that the solution of system (1) is stable and is heading for a disease-free equilibrium point $P_{1}\left(5 \times 10^{7}, 0,0,0\right)$. In other words, the disease-free equilibrium point $P_{1}\left(5 \times 10^{7}, 0,0,0\right)$ is locally asymptotically stable if $\Re_{0}<1$. The numerical simulation for this case supports the analysis results obtained in Theorem 1.
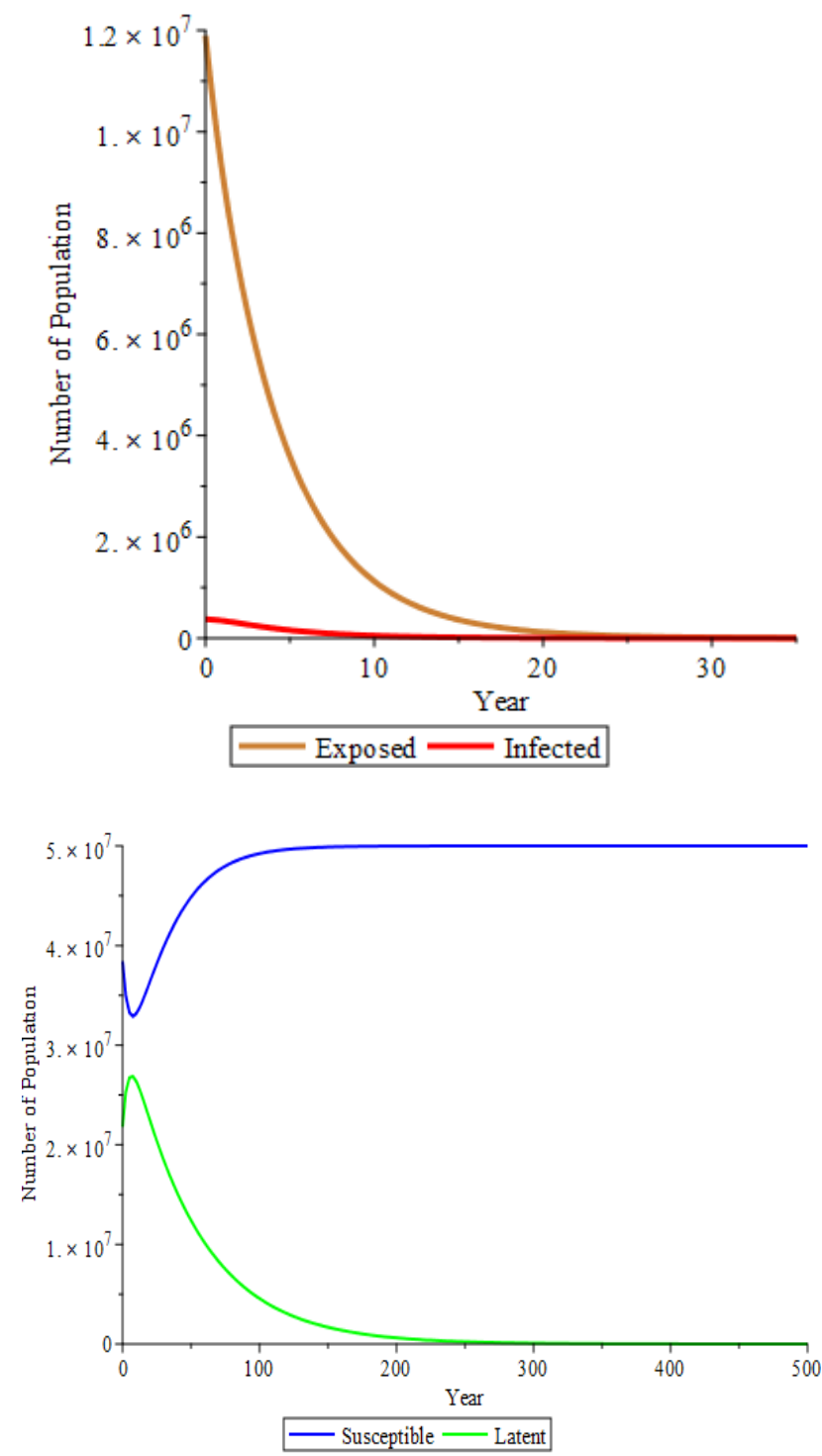

Figure 1. Graph SEIL of the model versus time for case $\Re_{0}<1$

The following are the parameters used to simulate the model in case $1<\Re_{0}<3$.

Table 2. Parameter Values for Cases $1<\mathfrak{R}_{0}<3$

\begin{tabular}{c|c|c}
\hline Parameter & $\begin{array}{c}\text { Estimated } \\
\text { (per year) }\end{array}$ & Source \\
\hline$b$ & 0,04 & (Kim et al., 2018) \\
$\beta$ & $1,6 \times 10^{-7}$ & (Mengistu \& Witbooi, 2019) \\
$\mu$ & 0,02 & (Kim et al., 2018) \\
$\alpha$ & 0,2 & (Kim et al., 2018) \\
$\gamma$ & 0,023 & (Mengistu \& Witbooi, 2019) \\
$p$ & 0,2 & (Kim et al., 2018)
\end{tabular}




\begin{tabular}{c|c|c}
$r$ & 0,546 & (Mengistu \& Witbooi, 2019) \\
$d$ & 0,05 & (Kim et al., 2018) \\
$K$ & $5 \times 10^{7}$ & Assumed \\
$S_{0}$ & $3,8 \times 10^{7}$ & (Mengistu \& Witbooi, 2019) \\
$E_{0}$ & $4 \times 10^{6}$ & Assumed \\
$I_{0}$ & $10^{5}$ & Assumed \\
$L_{0}$ & $2,18 \times 10^{7}$ & (Mengistu \& Witbooi, 2019)
\end{tabular}

Based on the values of the parameters in Table 2, we have $\mathfrak{R}_{0}=1,28$. The numerical solution of the system (1) for this case is given in the following graph.

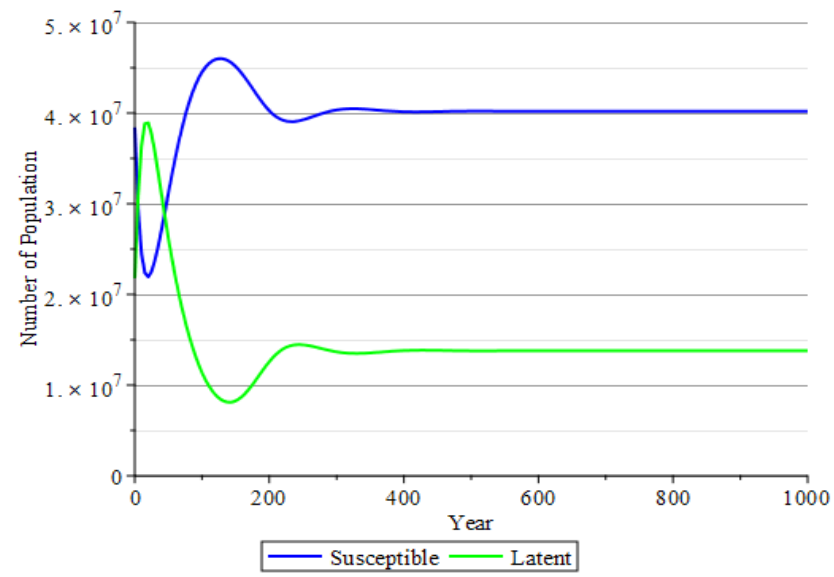

Figure 2. Graph $S(t)$ and $L(t)$ of the model versus time for case $1<\Re_{0}<3$

Figure 2 shows the number of population in the susceptible class $S(t)$ and the latent class $L(t)$ at first, there was a dynamic (fluctuating). However, for $t \rightarrow \infty$ the number of population in the two classes, respectively, approaches to $4 \times 10^{7}$ and $1,46 \times 10^{7}$ and is stable at that number.

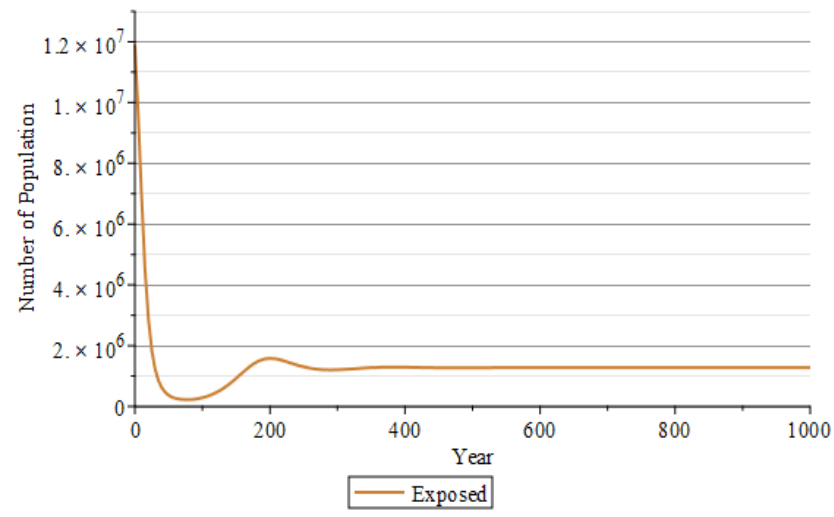

Figure 3. Graph $E(t)$ of the model versus time for case $1<\Re_{0}<3$
Figure 3 shows the population in the exposed class $E(t)$ at first, there was a dynamic (fluctuating). However, for $t \rightarrow \infty$ the number of people in that class approaches to $1,56 \times 10^{6}$ and is stable at that number.

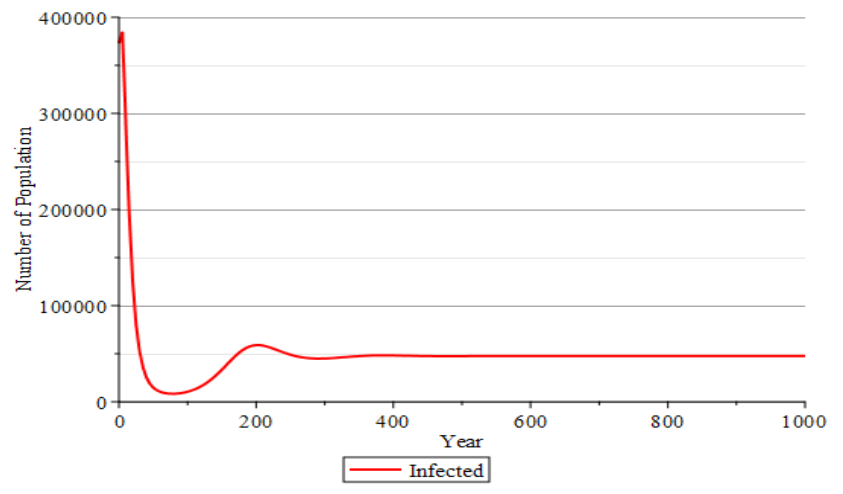

Figure 4. Graph $I(t)$ of the model versus time for case $1<\Re_{0}<3$

Figure 4 shows the population in the infected class $I(t)$ at first; there was a dynamic (fluctuating). However, for $t \rightarrow \infty$ the number of people in that class is getting to a number $5 \times 10^{4}$ and is stable at that number. It means that for the case $1<\Re_{0}<3$, the solution System (1) is stable and towards the endemic equilibrium point $P^{*}\left(4 \times 10^{7}, 1,56 \times 10^{6}, 5 \times\right.$ $\left.10^{4}, 1,46 \times 10^{7}\right)$. In other words, the endemic equilibrium point $P^{*}\left(4 \times 10^{7}, 1,56 \times 10^{6}, 5 \times 10^{4}, 1,46 \times 10^{7}\right)$ is locally asymptotically stable if $1<\mathfrak{R}_{0}<3$. The numerical simulation for this case supports the analysis results obtained in Theorem 2.

Table 3. Parameter Values for Cases $\mathfrak{R}_{0}>3$

\begin{tabular}{c|c|c}
\hline Parameter & $\begin{array}{c}\text { Estimated } \\
\text { (per year) }\end{array}$ & Source \\
\hline$b$ & 0,04 & (Kim et al., 2018) \\
$\beta$ & $1,6 \times 10^{-7}$ & (Mengistu \& Witbooi, 2019) \\
$\mu$ & 0,02 & (Kim et al., 2018) \\
$\alpha$ & 0,2 & (Kim et al., 2018) \\
$\gamma$ & 0,05 & Assumed \\
$p$ & 0,2 & (Kim et al., 2018) \\
$r$ & 0,29 & (Kim et al., 2018) \\
$d$ & 0,05 & (Kim et al., 2018) \\
$K$ & $5 \times 10^{7}$ & Assumed \\
$S_{0}$ & $3,8 \times 10^{7}$ & (Mengistu \& Witbooi, 2019) \\
$E_{0}$ & $1,19 \times 10^{7}$ & (Mengistu \& Witbooi, 2019) \\
$I_{0}$ & $3,73 \times 10^{5}$ & (Mengistu \& Witbooi, 2019) \\
$L_{0}$ & $2,18 \times 10^{7}$ & (Mengistu \& Witbooi, 2019) \\
\hline
\end{tabular}


Based on the values of the parameters in Table 3, we obtained $\Re_{0}=4,26$. The numerical solution of the system (1) for this case is given in the following graph.

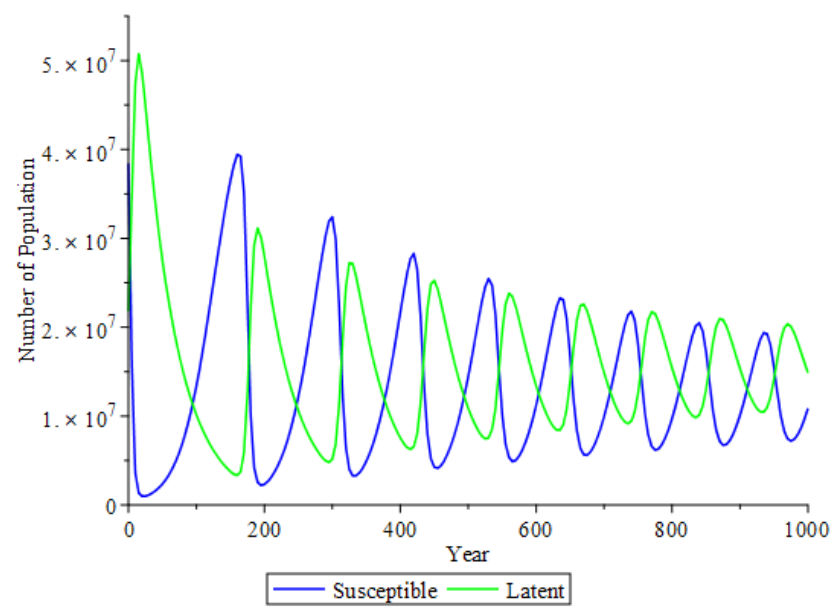

Figure 5. Graph $S$ and $L$ class versus time for the case $\mathfrak{R}_{0}>3$

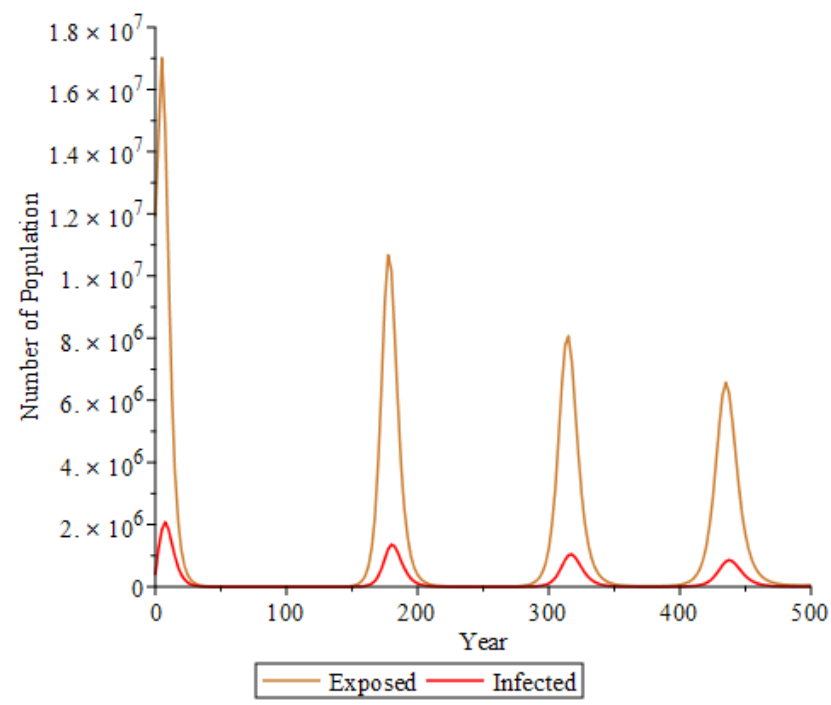

Figure 6. Graph $E$ and $I$ class versus time for the case $\Re_{0}>3$
Figure 6 shows that the population in the susceptible and the latent class oscillates to infinity. It means that the endemic equilibrium point $P^{*}$ is unstable for the case $\Re_{0}>3$.

\section{CONCLUSION}

Tuberculosis disease spread models involving logistical growth have been presented in this article. This model is the SEIL model in Kim2016 paper, which is modified to the system (1). Next, determine the equilibrium points and the basic reproduction number $\left(\Re_{0}\right)$ of the system (1). The result shows that equilibrium points in the system (1) depend on the basic reproduction number. If $\Re_{0}<1$, then there exist a unique equilibrium point, that is, the disease-free equilibrium point $P_{1}=(K, 0,0,0)$. Conversely, if $\Re_{0}>1$ there are two equilibrium points, the disease-free equilibrium point $P_{1}=(K, 0,0,0)$ and the endemic equilibrium point $P^{*}=$ $\left(S^{*}, E^{*}, I^{*}, L^{*}\right)$.

The local stability of the two equilibrium points is analysed using the Jacobian matrix. The results show that the diseasefree equilibrium point $P_{1}$ is locally asymptotically stable if $\Re_{0}<1$. While the endemic equilibrium point $P^{*}$ is locally asymptotically stable if $1<\Re_{0}<3$. The numerical simulation for each case shows a graph of the solution of the system (1). The results obtained from the numerical simulation support the analysis results that have been obtained.

\section{REFERENCES}

Castillo-Chavez, C \& Feng, Z 1997, 'To treat or not to treat: the case of tuberculosis.', Journal of Mathematical Biology, vol. 35 , no. 6 , pp. 629-656.

Castillo-Chavez, C \& Song, B 2004, 'Dynamical models of tuberculosis and their applications', Mathematical Biosciences and Engineering, vol. 1, no. 2, pp. 361-404. doi: 10.3934/mbe.2004.1.361.

Choi, S \& Jung, E 2014, 'Optimal tuberculosis prevention and control strategy from a mathematical model based on real data', Bulletin of Mathematical Biology, vol. 76, no. 7, pp.
1566-1589. doi: 10.1007/s11538-014-9962-6.

Driessche van den, P 2017, 'Reproduction numbers of infectious disease models', Infectious Disease Modelling, vol. 2, no. 3, pp. 288-303. doi: 10.1016/j.idm.2017.06.002. Jia, ZW, Tang, GY, Jin, Z, Dye, C, Vlas, SJ, Li, XW, Feng, D, Fang, LQ, Zhao, WJ \& Cao, WC 2008, 'Modeling the impact of immigration on the epidemiology of tuberculosis', Theoretical Population Biology, vol. 73, no. 3, pp. 437-448. doi: 10.1016/j.tpb.2007.12.007.

Jung, E, Lenhart, S \& Feng, Z 2002, 'Optimal control of 
treatments in a two-strain tuberculosis model', Discrete and Continuous Dynamical Systems - Series B, vol. 2, no. 4, pp. 473-482. doi: 10.3934/dcdsb.2002.2.473.

Kim, S, de los Reyes, AA \& Jung, E 2018, 'Mathematical model and intervention strategies for mitigating tuberculosis in the Philippines', Journal of Theoretical Biology, vol. 443, pp. 100-112. doi: 10.1016/j.jtbi.2018.01.026.

Li, J, Teng, Z, Wang, G, Zhang, L \& Hu, C 2017, 'Stability and bifurcation analysis of an SIR epidemic model with logistic growth and saturated treatment', Chaos, Solitons and Fractals, vol. 99, pp. 63-71. doi: 10.1016/j.chaos.2017.03.047.

Mengistu, AK \& Witbooi, PJ 2019, 'Modeling the effects of vaccination and treatment on tuberculosis transmission dynamics', Journal of Applied Mathematics, vol. 201, pp. 19. doi: 10.1155/2019/7463167.

Moualeu, DP, Weiser, M, Ehrig, R \& Deuflhard, P 2015, 'Optimal control for a tuberculosis model with undetected cases in Cameroon', Communications in Nonlinear Science and Numerical Simulation, vol. 20, no. 3, pp. 986-1003. doi: 10.1016/j.cnsns.2014.06.037.

Perko, L 2001, Differential Equations and Dynamical Systems. 3th edn. New York: Springer. Available at: https://www.springer.com/gp/book/9780387951164\#abo utBook.

Revelle, CS, Lynn, WR \& Feldmann, F 1967, 'Mathematical models for the economic allocation of tuberculosis control activities in developing nations', American Review of Respiratory Disease, vol. 96, no. 5, pp. 893-909. doi: 10.1164/arrd.1967.96.5.893.

Villasin, KJB \& Lao, AREMR 2017, 'A dynamical analysis of tuberculosis in the Philippines', Philippine Science Letters, vol. 10, no. 1, pp. 29-37. Available at: https://pdfs.semanticscholar.org/1cod/397ed2of9363a8d e40do84c19e1026a25d98.pdf.

Waaler, H, Geser, A \& Andersen, S 1962, 'The use of mathematical models in the study of the epidemiology of tuberculosis', American Journal of Public Health and The Nation's Health, vol. 52, no. 6, pp. 1002-1013. doi: 10.2105/AJPH.52.6.1002.

Whang, S, Choi, S \& Jung, E 2011, 'A dynamic model for tuberculosis transmission and optimal treatment strategies in South Korea', Journal of Theoretical Biology, vol. 279, no. 1, pp. 120-131. doi: 10.1016/j.jtbi.2011.03.009.

Zhao, Y, Li, M \& Yuan, S 2017, 'Analysis of transmission and control of tuberculosis in Mainland China, 2005-2016, based on the age-structure mathematical model', International Journal of Environmental Research and Public Health, vol. 14, no. 10, pp. 1-14. doi: $10.3390 /$ ijerph14101192. 